\title{
DEEP LEARNING TO DE-RISK RESERVE ESTIMATION
}

A PREPRint SUbMitTed TO CAMbRidge Open ENGAGE

\author{
(1) Yohei Nishitsuji \\ Department of Geoscience and Engineering \\ Delft University of Technology \\ Delft, The Netherlands \\ y.nishitsuji@tudelft.nl
}

\author{
(1) Shogo Masaya \\ Technical Division \\ INPEX \\ Tokyo, Japan \\ shogo.masaya@inpex.co.jp
}

July 15,2020

\begin{abstract}
Oil and gas companies evaluate the possibility of finding oil and gas fields carefully more than ever because it has been difficult to find gigantic discoveries which directly leads to their capital. Since a conventional evaluation contains human interpretation, luck and uncertainties, a variety of ranges of the reserves are often inferred from different interpreters given even identical dataset and conditions. As a consequence, there are differences between actual reserves and evaluated reserves. In this paper, using certain cases of how much actual reserves are deviated from interpreted reserves, deep learning is applied to mitigate such differences for unknown data which do not have actual reserves information. We find that our approach stably predicts the actual model by decreasing the misfit between the human and actual in comparison with the validation data on our workflow. The approach could be used to de-risk reserves estimation without changing traditional way of interpretations.
\end{abstract}

Keywords Deep learning $\cdot$ Hyperparameter optimization $\cdot$ Optuna $\cdot$ Oil\&Gas reserves prediction

\section{Introduction}

Discovering and developing commercial amount of hydrocarbon reserves is usually the ultimate task for oil and gas companies. When several companies evaluate certain prospects, namely expected reserves, a variety of the estimated reserves are often inferred because of the evaluation basically depends on interpreters. This is even valid when each interpreter has identical datasets and software applications at their hands. Milkov and Navidi [5] took a vast survey for 237 current industry practitioners and found that $90 \%$ of them believe that luck affects exploration projects. Moreover, their study revealed that to the variance in POS (Probability of Success) for actual results of exploration drilling around the world in 2008-2017 contributes not only individual perception but also luck with $39 \%$. This means that $39 \%$ of the variance in POS can not be distinguishable from randomness or serendipity. Thus, it is logical to state that human interpretations (experiences), which could be mixed with randomness and serendipity but cannot be distinguished from them, are mainly responsible for the cause of the above uncertainty [e.g., 5, 6].

From the aspect of management, such an heuristic approach or phenomenon is a far from an ideal situation for the related decision because it is impossible to objectively or quantitatively explain and judge for their outcomes, i.e., actual results of drills, after all. For the evaluation process to be quantitative, there are a few novel ideas which have been proposed. SAAM (Seismic Attribute Analysis Module) https://www.roseassoc.com/software-oil-gas-prospect-playportfolio/seismic-amplitude-analysis-module/is one of them aiming to overcome the weakness of POS or COS (Chance of Success) concepts. It includes quantitative seismic information into the notion of POS. Then, using the database of comparison between prediction and actual drills (over 340 cases as of September 2019), SAAM tries to improve its accuracy for next drills basically via the Bayes theorem.

In this study, we propose to use deep learning technology for reducing human interpretation for estimation of the expected reserves with quantitative prediction accuracy. We assume a situation where interpreters or estimation teams would like to utilize their standard for the evaluation process to a third party (i.e. new members) without deviating from their so-far established standard. In other words, the deep learning would mitigate the mismatch between the expected 
reserves, based on their "company standard", and the actual results. We show how to implement this approach and demonstrate its effectiveness with synthetic examples.

\section{Method and Synthetic Dataset}

In Figure 1 a schematic view of our proposed workflow is shown. The deep-learning architecture, which is a part of the workflow (Figure 1], is shown separately in Figure 2. We use fully connected layers [e.g., 4] as deep-learning model.

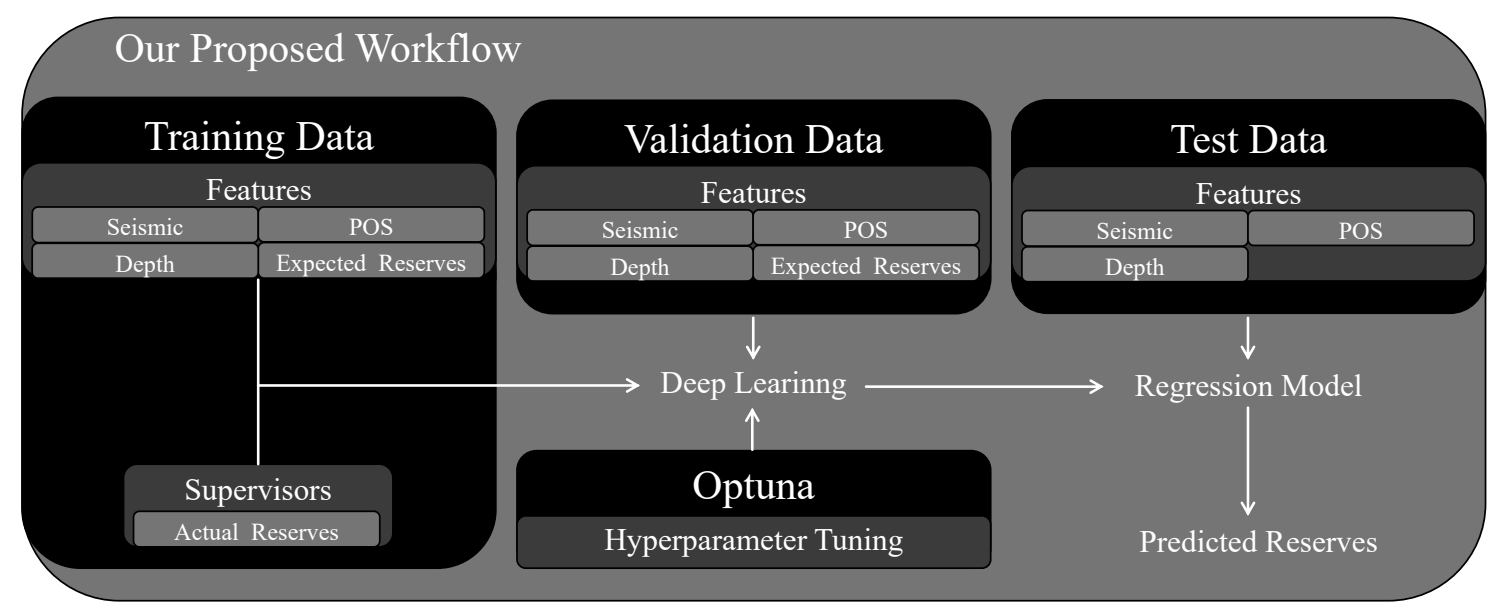

Figure 1: A schematic workflow of this study. An architecture of the used deep learning is separately depicted in Figure 2.

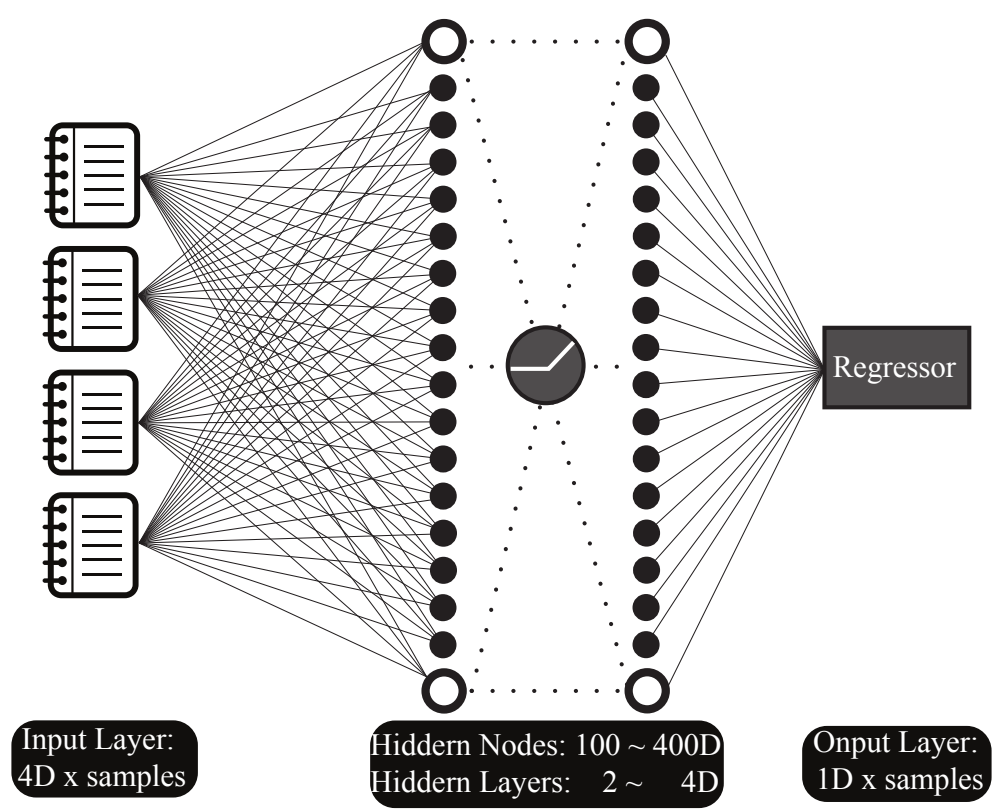

Figure 2: Used fully connected layer deep-learning model in this study. The total number of hidden nodes (100 to 400), hidden layers (2 to 4 ), dropout rate (0 to 1), learning rate (0.01 to 0.00001) and a solver of loss function (Adam or RMSprop)[e.g., 8] are optimized by the global optimizer Optuna [1]. The clock-like icon stands for an activator - a rectified linear unit.

One of the drawbacks in the standard deep-learning approach is that several of their hyperparameters (e.g., dropout rate and learning rate) need to be optimized to improve the prediction accuracy. To overcome this, an automatic optimization 
framework called Optuna [1] is adapted in our proposed method. Optuna is a powerful framework which provides an optimum combination of hyperparameters with relatively lower computation cost in comparison with other optimization schemes like exhausted grid search and random grid search [1].

Apart from the model design, a choice of an input dataset is of importance. To obtain an approach that minimizes the bias caused by human interpretation, i.e., a fully data-driven approach, the amount of available data should be adequate to explain and find hidden relationship or patterns between the data and given targets. For our case, the data and the targets can be derived from seismic and well dataset. As explained above, however, we assume the situation of transferring or utilizing the established standard (say, company standard) of estimating the reserves to others. Hence, what we need in practice are namely POS, some physical data (e.g., depth), some categorical parameters (e.g., region names if a variety of ranges is available) and associated actual result of drills.

To make the above condition computationally viable, we make 500 synthetic datasets with uniform-random parameters consisting of seismic information, POS, depth, expected reserves, and actual reserves. For the sake of practical application of deep learning, the values of the seismic information, depth and expected reserves are normalized between 0 and 1 . Here, the seismic information is defined by all of the information related to seismic data quality, including acquisition and processing quality, and interpretation, such as direct hydrocarbon indicator (DHI) and/or amplitude-versus-offset analysis.

Our calculation is based on a hypothetical situation. Since we would like to test how deep learning corrects mismatch between the expected and actual reserves, we generate an artificial mismatch between them on purpose. Then, we use deep learning to automatically and appropriately correct that mismatch for new data. For convenience, we let the depth information not relate to the expected reserves. In this synthetic data experiment, a pseudo relationship to generate the expected reserves is assumed:

$$
\text { Expected Reserves }=2^{(\text {Seismic*weight\#1+POS*weight\#2)},}
$$

where weight\#1 and weight\#2, which are arbitrary integers, are set to be 13 and 4 to make the expected reserves be around between 0 and 70 million boe (barrel of oil equivalent). On the other hand, as a hypothetical task, we intentionally assume that actual reserves can be derived from other mathematical expressions, thus generating the mismatch. That is, we obtain the following expression:

$$
\text { Actual Reserves }=2^{(\text {Seismic*weight\#3+POS*weight\#4+depth*weight\#5)},}
$$

where weight\#3, weight\#4, and weight\#5 are arbitrary integers. In our case, to make the actual reserves be around between 0 and 70 million boe, we choose 13,1 and 3 for weight\#3, weight\#4, and weight\#5, respectively. While equation 1 can be seen as the company or individual experience, equation 2 stands for what the actual results are. Therefore, the difference between equations 1 and 2 is now simplified to a task minimize their difference by keeping equation 2 .

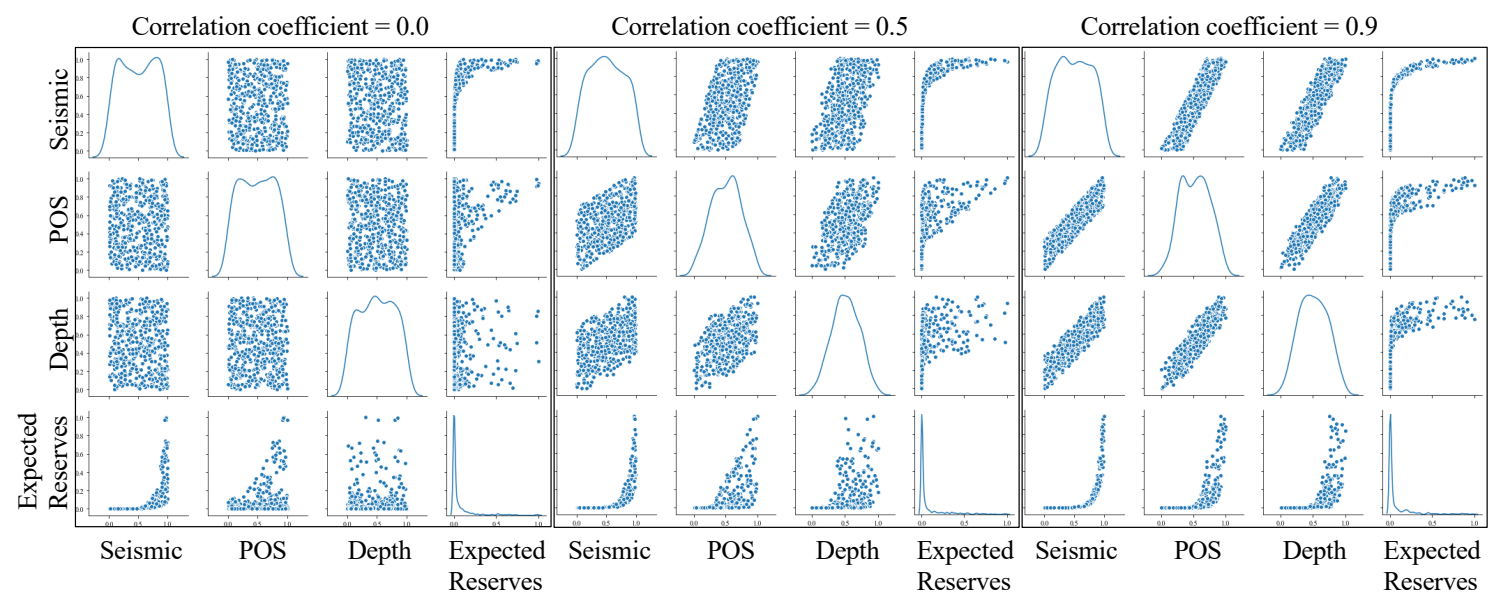

Figure 3: A view of the synthetic data when the correlation is set to be $0.0,0.5$, and 0.9 . 
The magnitude of correlation among the seismic, POS and depth information is arbitrary or up to the established standards. Here, we set up several situations for the correlation ranging from 0.0 (completely random) to 0.9 (strong correlation) using the so-called Cholesky factorization (Figure 3), which is a decomposition of a Hermitian, positivedefinite matrix into the product of a lower triangular matrix and its conjugate transpose for efficient numerical solutions. It can be noted that the expected reserves appear as a log-normal distribution with their mean around 0 . This appearance is similar to what the actual oil and gas distributions are found to be [e.g., 2, 7], which is what we would like to synthesize. For generating the synthetic dataset, normal random parameters are also tested but not used because the distribution of the expected reserves derived from equation 1 turned out to be jaggy as a whole.

An example of 5 out of the 500 samples when correlation is 0.0 is shown in Table 1 . We then split the 500 synthetic samples into 300 training, 100 test, and 100 validation data. The number of training and validation data are equally split for an evaluation purpose to be described later. After finding the best observed hyperparameters, those parameters are used for the test data to predict the actual reserves. The number of epochs is set to be 800 .

\begin{tabular}{|c|c|c|c|c|c|}
\hline $\begin{array}{l}\text { Normalized } \\
\text { Seismic }\end{array}$ & POS & $\begin{array}{l}\text { Normalized } \\
\text { Depth }\end{array}$ & $\begin{array}{l}\text { Expected } \\
\text { Reserves } \\
\text { (mmboe) }\end{array}$ & $\begin{array}{l}\text { Actual } \\
\text { Reserves } \\
\text { (mmboe) }\end{array}$ & $\begin{array}{l}\text { DL } \\
\text { Prediction } \\
\text { (mmboe) }\end{array}$ \\
\hline 0.26 & 0.94 & 0.33 & 156 & 43 & $?$ \\
\hline 0.76 & 0.32 & 0.07 & 2174 & 1298 & $?$ \\
\hline 0.81 & 0.68 & 0.09 & 10660 & 3122 & ? \\
\hline 0.35 & 0.07 & 0.51 & 30 & 75 & ? \\
\hline
\end{tabular}

Table 1: Synthesized input parameters for simulation. Assuming they are completely independent each other, 5 out of 500 samples are shown. Expected reserves and actual reserves are both mathematically formulated using different equations to make an intentional mismatch to be later corrected by the deep-learning technology. These vales (thus except for the actual reserves) used in the training are to be normalized.

\section{Results and Discussion}

Figure 4 shows 50 learning curves evaluated by Optuna. We find that the early trails (tending towards blue color) are more completed till the end of epochs while the later trials (tending towards red color) are pruned as being expected due to the criteria based on the median value in the past trails, which is an effect provided by Optuna. The loss generally decreased as a function of epoch, which indicates that the process at least is not overfitting. The best values of each of the hyperparameters estimated in Figure 4 are passed to the actual prediction phase as shown in Figure 5.

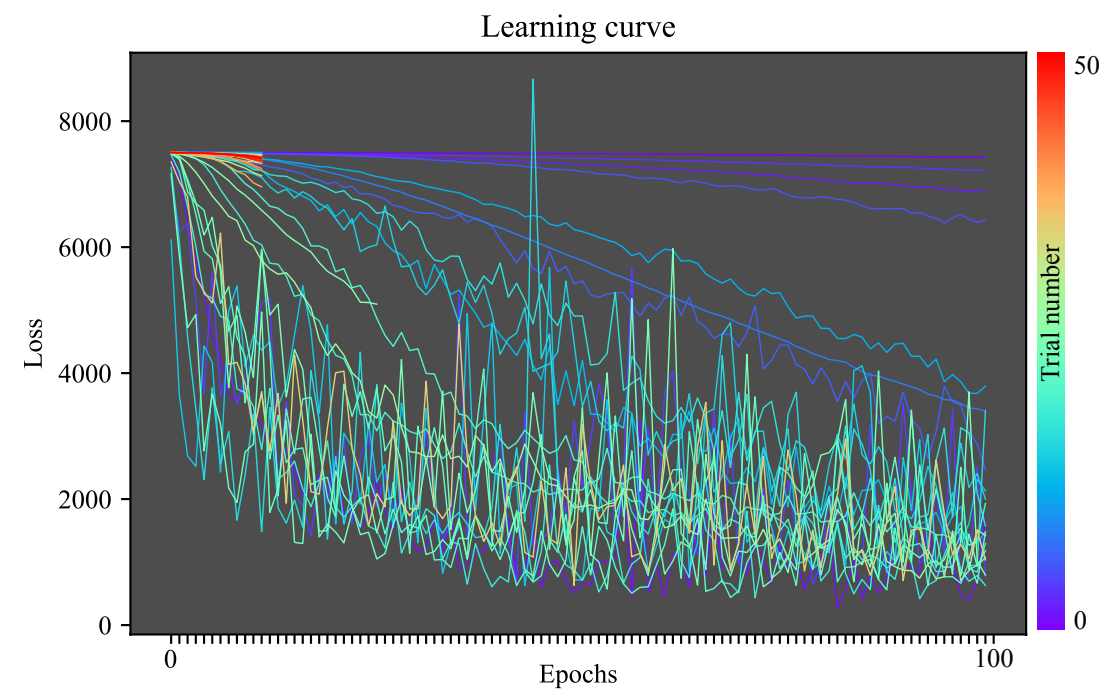

Figure 4: 50 learning curves computed by Optuna with each up to 100 epochs when the correlation coefficient of the input parameters is 0.5 . The entire learning process is not necessarily completed if the intermediate value (of loss) is less than the median values obtained so far. Minimum number of epochs is set to be 10 . 


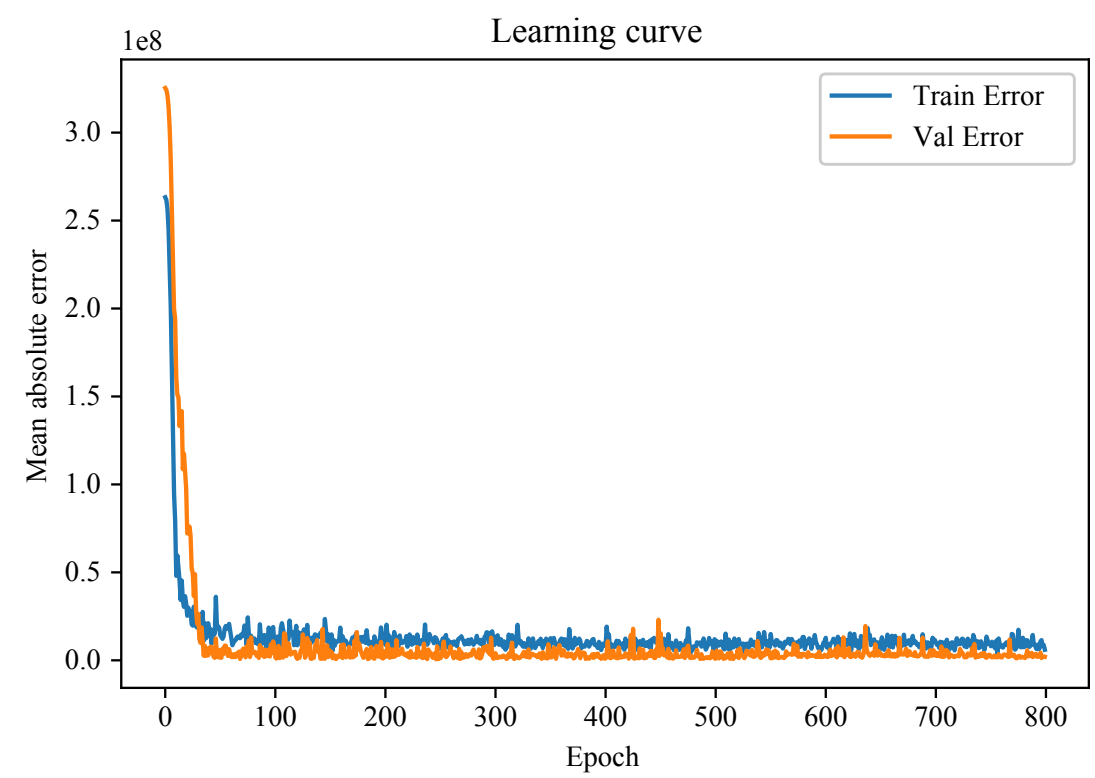

Figure 5: Learning curves using hyperparameters from Figure 4. Both learning curves for training (train) and validation (val) data are shown.

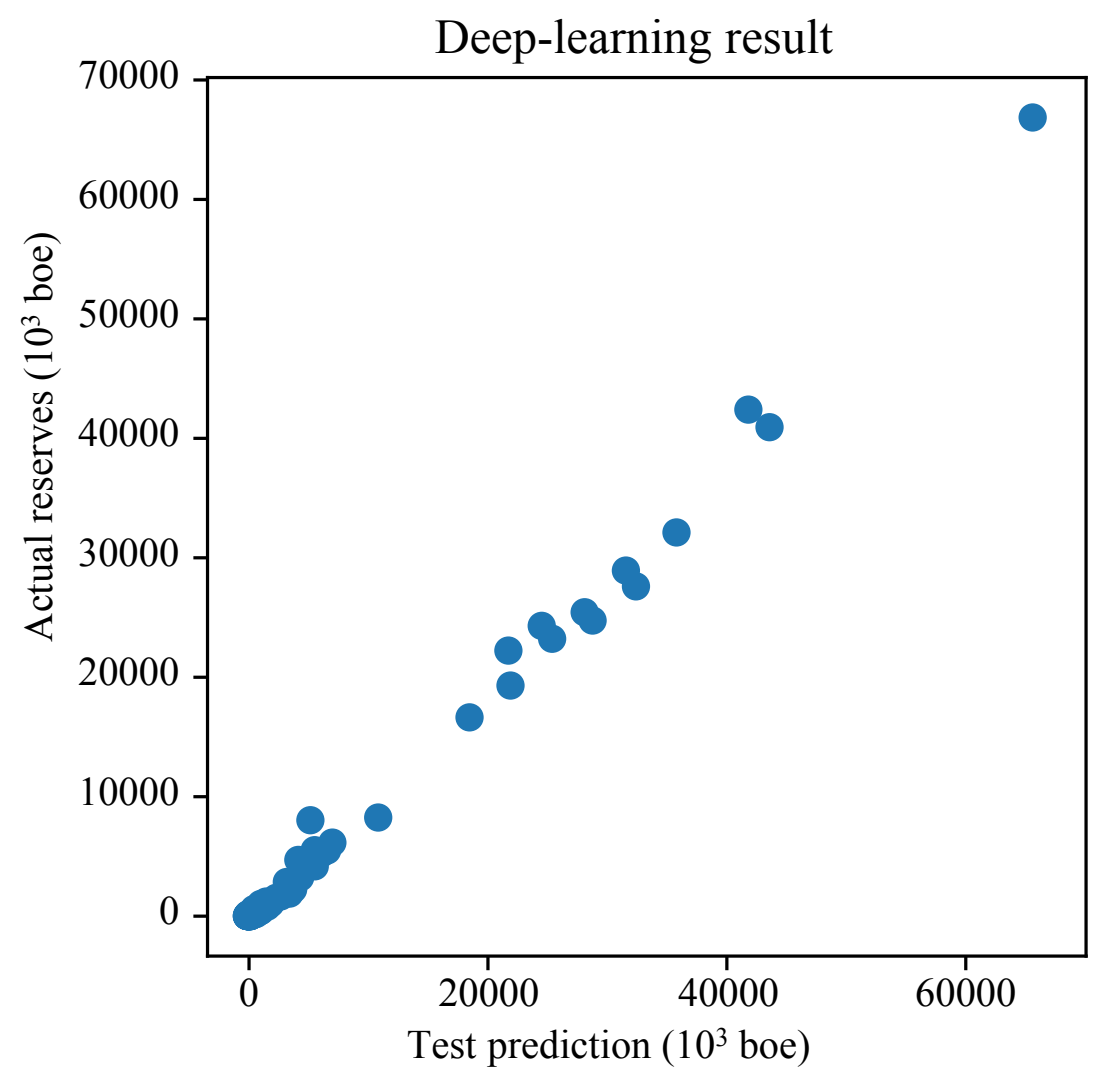

Figure 6: The prediction of the actual reserves using the optimized model from Figure 5 where the correlation coefficient of the input parameters is set to be 0.5 . 
As being implied by Figures 4 and 5 , the learning curves of both training and validation data turn out to be stable. Given this optimized deep-learning model, we predict the actual reserves in comparison with their ground truth derived by equation 2. Including the largest reserve, the prediction appears to work stably as can be seen in Figure 6 .

In our study, we used Optuna to efficiently optimize representative hyperparameters of the fully connected type of deep learning. In case of not adapting the pruning function embedded in Optuna, which should be equivalent to other sampling framework such as Hyperopt [3], we found the computation cost increases 1.7 times by using the example of Figure 4 In terms of the misfit value, Hyperopt should provide optimized hyperparameters at least as good as the one given by Optuna. However, it is difficult to quantitatively ascertain how much the misfit would be with and without the pruning because we cannot reproduce an exactly identical situation between both.

To validate whether our method can be stably applied when different correlation coefficients are given between the input parameters, we plot the misfit between the actual and the predicted reserves as a function of the correlation coefficient. The result is shown in Figure 7

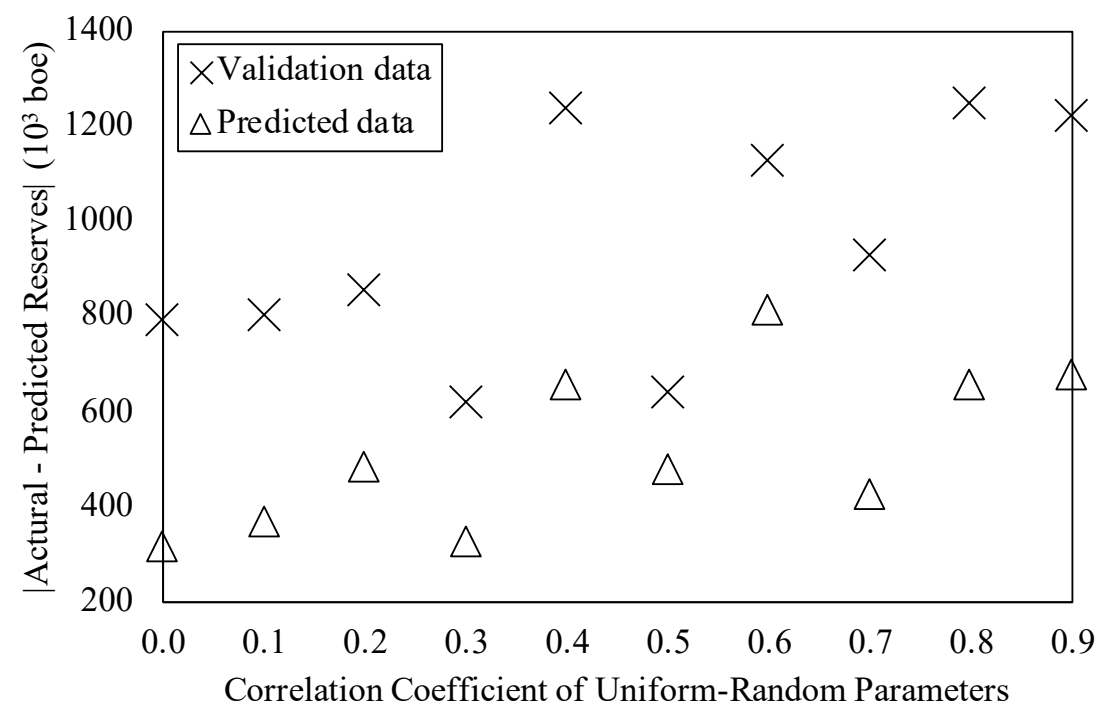

Figure 7: Absolute differences between the actual and predicted reserves plotted for correlation coefficients from 0.0 to 0.9 with a step of 0.1 .

Since we do not know how many trails for Optuna is quantitatively sufficient, it is difficult to define what the best hyperparameters are (Figure 47. However, assuming that 50 of the trails are adequate in our study, we find that the mismatch between the prediction and the actual reserves, which is responsible to human bias and luck, was stably decreased in comparison with the validation data regardless of the correlation coefficient used to synthesize data.

As future tasks to further validate our model rigorously, an application to real dataset or different synthetic data derived from other randomizers and/or equations instead of equations 1 and/or 2 could be considered.

\section{Conclusions}

We demonstrated how we minimize the difference between the expected reserves and the actual reserves by deeplearning technology with the global-optimizer scheme. We found that our approach stably predicts the actual model without overfitting. Moreover, the misfit between the model and the ground truth is decreased in comparison with the validation data regardless of the correlation coefficient used to synthesize the data. Our model could be used to shift the expected reserves to the actual reserves without changing company or individual standards.

\section{Acknowledgments}

The authors thank Deyan Draganov and Lukas Mosser for their assistance of this submission. For synthetic computation of deep learning, we use TensorFlow, Keras and Optuna. 


\section{References}

[1] T. Akiba, S. Sano, T. Yanase, T. Ohta, and M. Koyama. Optuna: a next-generation hyperparameter optimization framework. In Proceedings of 25th ACM SIGKDD Conference on Knowledge Discovery and Data Mining, page 2623-2631, Anchorage, 2019. SIGKDD.

[2] J. J. Arps and T. G. Roberts. Economics of drilling for cretaceous oil on east flank of denver-julesburg basin. AAPG Bulletin, 42, 1958.

[3] J. Bergstra, B. Komer, C. Eliasmith, D. Yamins, and D. D. Cox. Hyperopt: a python library for model selection and hyperparameter optimization. Computational Science \& Discovery, 8, 2015.

[4] L.-C. Chen, A. G. Schwing, A. L. Yuille, and R. Urtasun. Learning deep structured models. In Proceedings of the 32nd International Conference on Machine Learning, page 1785-1794, Lille, 2015. JMLR.

[5] A. V. Milkov and W. C. Navidi. Randomness, serendipity, and luck in petroleum exploration. AAPG Bulletin, 104, 2020.

[6] D. Polson and A. Curtis. Dynamics of uncertainty in geological interpretation. Journal of the Geological Society, 167, 2010.

[7] D. G. Quirk and R. Ruthrauff. Analysis of reserves discovered in petroleum exploration. Journal of Petroleum Geology, 29, 2006.

[8] A. C. Wilson, R. Roelofs, M. Stern, N. Srebro, and B. Recht. The marginal value of adaptive gradient methods in machine learning. In Proceedings of the Advances in Neural Information Processing Systems 30, pages 4148-4158, California, 2017. NIPS. 